\title{
Detection and molecular characterization of Hepatozoon canis, Babesia vogeli, Ehrlichia canis, and Anaplasma platys in dogs from Metro Manila, Philippines
}

\author{
Davin Edric V. Adao ${ }^{1,2}$, Charles Michael T. Herrera ${ }^{1}$, Luiza H. Galarion ${ }^{1,2}$, Nicole R. Bolo ${ }^{1,2}$, Rhodora S. Carlos ${ }^{3}$, \\ Enrique T. Carlos ${ }^{4}$, Sixto S. Carlos ${ }^{4}$, Windell L. Rivera ${ }^{1,2, *}$ \\ ${ }^{1}$ Institute of Biology, College of Science, and ${ }^{2}$ Molecular Protozoology Laboratory, Natural Sciences Research Institute, \\ University of the Philippines Diliman, Quezon City 1101, Philippines \\ ${ }^{3}$ Carlos Veterinary Clinic, Parañaque City 1700, Philippines \\ ${ }^{4}$ Makati Dog and Cat Hospital, Makati City 1210, Philippines
}

(Received: January 19, 2017; Revised: March 24, 2017; Accepted: April 21, 2017)

\begin{abstract}
The study of canine vector-borne diseases in the Philippines started in the 1970s but only gained interest in the past decade. Characterization of such diseases in the Philippines remains incomplete, thus, it is necessary to obtain additional information on the prevalence and diversity of canine tick-borne diseases in the country. In this study, blood samples were obtained at two veterinary clinics in Metro Manila, Philippines from 114 dogs suspected of having canine tick-borne pathogens. Polymerase chain reaction (PCR) was performed on whole blood DNA extracts followed by sequencing, and the following pathogens were detected: Hepatozoon $(H$.) canis $(5.26 \%)$, Babesia $(B$.) vogeli $(5.26 \%)$, Ehrlichia (E.) canis (4.39\%), and Anaplasma platys (3.51\%). Additionally, a set of multiplex PCR primers were developed to detect H. canis, Babesia spp. (B. canis and B. vogeli), and E. canis in canine blood. Multiplex and conventional single-reaction PCR results for the 114 dog blood samples were similar, except for one $H$. canis sample. Multiplex PCR is, therefore, a useful tool in screening infected dogs in veterinary clinics. This study's results, together with those of previous studies in the country, show that canine vector-borne pathogens are an emerging veterinary concern in the Philippines.
\end{abstract}

Keywords: Philippines, canine vector-borne diseases, dogs, molecular detection, multiplex polymerase chain reaction

\section{Introduction}

Canine vector-borne diseases (CVBDs) caused by the protozoan parasites Hepatozoon spp. and Babesia spp. as well as members of the prokaryotic group Anaplasmataceae (e.g., Ehrlichia spp. and Anaplasma spp.) have been the subject of studies over the years due to their impact to both companion and working dogs [25]. Once canine vector-borne (CVB) infections manifest in canine blood cells, subclinical (e.g., lethargy, depression) to potentially fatal (e.g., multiple organ failure) symptoms can occur. The causative agents of CVBDs are transferred via tick vectors, hence, the travel and settlement of domestic dogs to areas where these vectors and pathogens are endemic (e.g. near wilderness areas) have contributed to their spread $[25,29]$. In the Philippines, there were already reports of CVBDs in humans and dogs in the 1970s. The presence of Hepatozoon sp. in a human patient [7] and in canines [24] as well as Babesia sp. in canines [6] has been reported. However, follow-up studies were only available decades later with the advent of molecular meth- ods leaving a large gap on surveillance of CVB infections for roughly three decades. These recent studies used polymerase chain reaction (PCR) detection to identify Hepatozoon $(H$.) canis, Anaplasma (A.) platys, Ehrlichia (E.) canis, and Babesia spp. in dogs [2, 8, 36, 37, 39] and in brown ticks (Rhipicephalus [R.] sanguineus) isolated from dogs [38]. While it is difficult to determine whether CVBDs were already endemic to the Philippines or brought by infected ticks or dogs from other countries, these studies proved that they are indeed present in domestic dogs and ticks, and that there is a need for additional prevalence data on CVBDs in the country.

This study aimed to survey the presence of Babesia spp., Hepatozoon spp., and Anaplasmataceae infecting domestic dogs in Metro Manila, Philippines and develop a multiplex PCR protocol that can be used to detect the presence of $H$. canis, Babesia spp. (Babesia [B.] canis and B. vogeli), and E. canis in dogs. The current conventional method of detection of CVBDs is blood smear examination (with Giemsa staining) together with observation of clinical signs $[1,15]$. However, this method poses several disadvantages. First, microscopy

\footnotetext{
*Corresponding author

Tel/Fax: +63-2-4266659

E-mail: wlrivera@science.upd.edu.ph
} 
is both labor-intensive and time-consuming. It also requires trained microscopists since infected cells can be confused with other blood cells such as platelets and white blood cells with phagocytosed materials [23]. Second, several clinical signs - such as fever, anorexia, depression, thrombocytopenia, and anemia - are common to CVB infections, and may vary among hosts [32]. Finally, concurrent infections - which are not easily detected in microscopy - are known to occur as some of these pathogens have the same tick vector (e.g. E. canis, $H$. canis, and B. vogeli are all carried by $R$. sanguineus) [29]. If these difficulties are ignored, misdiagnosis of the disease can occur. Thus, multiplex PCR is an affordable, accurate, and rapid method of detection that can be used for proper detection of CVBDs. Ultimately, investigation of prevalence and efficient screening of the pathogens are important first steps in controlling the spread of CVBDs, most especially in the Philippines where inter-island transmission can lead to infection of local populations lacking immunity against CVBDs.

\section{Materials and Methods}

\section{Sample collection}

The research protocol used in this study was reviewed and approved by the Institutional Animal Care and Use Committee of the University of the Philippines Los Baños under assigned protocol no. 2012-35. A total of 114 canine blood samples were obtained from the Makati Dog and Cat Hospital in Makati City, Philippines and the Carlos Veterinary Clinic in Parañaque City, Philippines from 2013-2014. All of these dogs were suspected to have CVBDs due to presence of signs of infection such as high fever $\left(>39^{\circ} \mathrm{C}\right)$, low platelet count $\left(<200,000\right.$ platelets $\left./ \mathrm{mm}^{3}\right)$, low white blood cell count $\left(<6,000\right.$ white blood cells $\left./ \mathrm{mm}^{3}\right)$, presence of clinical signs (e.g., lethargy), presence of ticks, and positive serology results for Ehrlichia sp. DNA was extracted using DNAzol $\mathrm{BD}$ reagent (Life Technologies; USA) following the manufacturer's protocol, and extracts were stored at $-4^{\circ} \mathrm{C}$ until PCR amplification.

\section{Molecular detection of $\boldsymbol{H}$. canis, Babesia spp., and Anaplasmataceae}

Conventional PCR and DNA sequencing using primers that are specific for target CVB pathogens were done. $H$. canis infections were identified by amplification and sequencing of $660 \mathrm{bp}$ PCR products using Hep-F/Hep-R [13]. Babesia spp. (B. canis, B. vogeli, and B. rossi) infections were identified by amplification and sequencing of PCR products using the primers PIRO-A1/PIRO-B, which produce a 450 bp PCR product [9]. Anaplasmataceae were detected and identified by amplification and sequencing of the $345 \mathrm{bp}$ products of the primers EHR16SD/EHR16SR $[12,26]$. Further identification of Anaplasma spp. was conducted by sequencing larger parts of the $16 \mathrm{~S}$ rRNA gene using the primer pairs fD1/EHR16SR and EHR16SD/Rp2 [12]. PCR amplification was performed using previously published protocols for Hep-F/Hep-R [16], PIRO-A1/PIRO-B [5], and EHR16SD/EHR16SR [34]. Full 16S rRNA gene amplification of Anaplasma spp. was performed according to the protocol of Inokuma et al. [12]. The primers Hep-F/Hep-R and PIRO-A1/PIRO-B target the18S rRNA genes of their respective CVB pathogens while primers EHR16SD, EHR16SR, fD1, and Rp2 amplify the 16S rRNA gene of Anaplasmataceae. PCR was performed thrice on positive samples and sent to the Philippine Genome Center for sequencing to rule out false positives. Sequences were aligned using the Clustal W feature of BioEdit 7.2.0 [11]. Sequences were then uploaded onto the nucleotide BLAST website (National Center for Biotechnology Information, USA) to determine the most similar sequences and confirm their species identity.

\section{Phylogenetic analysis}

Phylogenetic trees were constructed to confirm species identification of the samples. Neighbor joining (NJ) and maximum parsimony (MP) phylogenetic trees were constructed using either PAUP* 4.01b [31] or MEGA 6 software [33]. Maximum likelihood (ML) trees were constructed by uploading the aligned sequences to the PHYML website [10].

The phylogenetic tree of partial $H$. canis $18 \mathrm{~S}$ rRNA gene was constructed with sequences from $H$. canis (GenBank accession nos. EU289222 and KJ634654; National Center for Biotechnology Information), H. americanum (GenBank accession no. AF176836), H. catesbianae (GenBank accession nos. AF176837 and AF130361), H. felis (GenBank accession nos. KM435071, KC138533, and KC138534), and Sarcocystis arctosi (GenBank accession no. EF564590) as outgroup. The phylogenetic tree of Babesia spp. partial 18S rRNA gene was constructed with sequences from B. canis (GenBank accession nos. AY072926, AY270247, AY527063, AY649326, AY962186, AY962187, HQ662634, KC593877-KC593879, KF499115, KM111282, and KM111283), B. rossi (GenBank accession nos. AB303071-AB303075, AB935163-AB935166, DQ111760, JQ613104, JQ613105, and L19079), B. vogeli (GenBank accession nos. AB303076, AY072925, AY07771, DQ297390, HM590440, HQ148663, HQ148664, HQ662635, JN713120-JN713122, JX304677, JX304679-JX304683, JX112785, JX871891, KF970926, and KF970929), and Babesia sp. 'Spanish dog' (GenBank accession no. EU583387) with Babesia sp. 'Oklahoma dog' (GenBank accession no. AF20563) and B. gibsoni (GenBank accession no. KJ142323) as outgroups. The phylogenetic tree of Anaplasma sp. 16S rRNA gene was constructed with the sequences of $A$. bovis (GenBank accession nos. AB196475 and AB211163), A. centrale (GenBank accession nos. AB211164 and AF309869), A. marginale (GenBank accession nos. AF309867, AF309868, KU686788KU686793, and NR074556), A. odocoilei (GenBank accession no. JX876644), A. ovis (GenBank accession no. AY262124), A. phagocytophilum (GenBank accession nos. AB196720, AB196721, DQ458805, and DQ458808), A. platys (GenBank accession nos. AF536828 and EU439943), Anaplasma sp. 
(GenBank accession nos. KR261620-KR261622), and uncultured Anaplasma sp. (GenBank accession nos. JN862824 and JX402624) with E. canis (GenBank accession no. U26740) as an outgroup.

\section{Multiplex primer set design}

Several gene sequences of E. canis, B. canis, B. vogeli, B. rossi, and $H$. canis were obtained from the GenBank Nucleotide Database for multiplex primer design. These primers were designed from $E$. canis $16 \mathrm{~S}$ rRNA gene sequences (GenBank accession nos. AB723707, AB723708, AB723709, AB723710, AB723711, AB723712, JX261981, and JX893522), $B$. canis, $B$. rossi, and $B$. vogeli $18 \mathrm{~S}$ rRNA gene sequences (GenBank accession nos. B. canis canis EU622792, B. canis canis AY072926, B. canis JF461263, B. canis rossi JQ613104, B. canis rossi JQ613105, B. canis canis JX227980, B. canis JX678979, B. canis vogeli JX871889, and B. canis vogeli JX871891), and $H$. canis 18S rRNA gene sequences (GenBank accession nos. AF176835, AY461378, JX441117, JX466885,

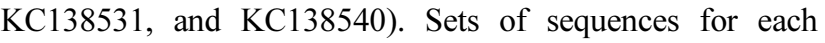
organism were aligned using the Clustal W feature of BioEdit 7.2.0 to determine regions specific for each of the target species. Gene sequences that had these conserved regions for each species were imported to PrimerPlex 2.6.2 (PREMIER Biosoft, USA). Multiplex primer sets specific for the gene sequences were then designed with primer pairs having melting temperatures within 55 to $65^{\circ} \mathrm{C}$. The designed primers targeted conserved regions of $E$. canis $16 \mathrm{~S}$ rRNA gene (Ehr16S_multiF and Ehr16S_multiR), H. canis 18S rRNA gene (Hep18S_multiF and Hep18S_multiF), and Babesia spp. (B. canis and B. vogeli) 18S rRNA gene (Bab18S_multiF and Bab18S_multiR) (Table 1). Amplicon sizes were restricted to 200 to $700 \mathrm{bp}$ in size at 100 to $150 \mathrm{bp}$ intervals. Default software specifications to avoid formation of secondary structures such as hairpin loops and primer dimers were used. Primer sequences were tested for specificity using both nucleotide BLAST (blastn) and primer BLAST features available at the National Center for Biotechnology Information (NCBI) website. The optimal multiplex primer set was then picked based on similarities in melting temperature, low tendency of secondary structure formation, and high specificity for target gene sequence.
Optimum annealing temperature and analytical sensitivity and specificity

Multiplex PCR was initially performed on positive control DNA extracts of E. canis, B. canis, and H. canis to obtain the optimum annealing conditions as well as analytical specificity and sensitivity of the designed primers. DNA extracts of positive controls were requested from the following universities: E. canis from the Koret School of Veterinary Medicine of the Hebrew University of Jerusalem (Israel), B. canis from the Department of Parasitology and Zoology of the Szent Istvan University (Godollo, Hungary), and $H$. canis from the Dipartimento di Medicina Veterinaria of the Universita degli Studi di Bari Aldo Moro (Bari, Italy). A. platys and B. vogeli DNA extracts were obtained during sampling of canine blood in this study. All multiplex PCR experiments were performed with KAPA2G Fast multiplex kit according to the manufacturer's protocol with $0.25 \mu \mathrm{M}$ of each multiplex primer. The optimum annealing temperature was obtained using gradient PCR $\left(58,59,60,61\right.$, and $62^{\circ} \mathrm{C}$ in each) on DNA extracts of E. canis, $B$. canis, and $H$. canis. Single reaction PCR was performed on DNA extracts of E. canis, $B$. canis, $H$. canis, B. vogeli and $H$. canis mixture, and $A$. platys to determine possible cross-reactions of primers with other canine blood-borne pathogens. Multiplex PCR was also performed on DNA extracts of Escherichia coli, Pseudomonas aeruginosa, Bacillus subtilis, and Staphylococcus aureus to determine if the primers would cross-react to any of these four common bacteria that may contaminate blood samples during blood or DNA extraction [3, 28]. Analytical sensitivity was determined by performing multiplex PCR on 1:10, $1: 100$, and 1:1,000 dilutions of available E. canis $(24.4 \mathrm{ng} /$ $\mu \mathrm{L})$, B. canis $(19.1 \mathrm{ng} / \mu \mathrm{L})$, and $H$. canis $(45 \mathrm{ng} / \mu \mathrm{L})$ DNA. Single or multiplex PCR mixes containing only the respective primers and no DNA template served as negative controls for all reactions.

\section{Results}

\section{Symptoms of CVBD in dogs sampled}

Of the 114 dogs sampled, $72(63.16 \%)$ presented only one sign of infection, $37(32.46 \%)$ presented a combination of any two signs of infection, while the five remaining dogs presented three signs of infection (4.39\%). The most com-

Table 1. Multiplex primers designed using PrimerPlex 2.6.2 (PREMIER Biosoft) that target the 18S rRNA gene regions of Hepatozoon canis (Hep) and Babesia spp. (B. canis and B. vogeli; Bab) and the 16S rRNA gene region of Ehrlichia canis (Ehr)

\begin{tabular}{|c|c|c|c|c|}
\hline Primer name & Sequence $\left(5^{\prime}\right.$ to 3 ') & $\mathrm{T}_{\mathrm{m}}\left({ }^{\circ} \mathrm{C}\right)$ & Target & Product size \\
\hline Ehr16S_multiF & 5'-GCCTAACACATGCAAGTCGAACGGACAATT-3' & 65.3 & \multirow{2}{*}{ Ehrlichia canis } & \multirow{2}{*}{$664 \mathrm{bp}$} \\
\hline Ehr16S_multiR & 5'-AACCAGATAGCCGCCTTCGCCACT-3' & 64.8 & & \\
\hline Bab18S_multiF & 5'-ACCCAAACCCTCACCAGAGTAGCAATT-3' & 63 & \multirow{2}{*}{$\begin{array}{l}\text { Babesia spp. } \\
\text { (B. canis and B. vogeli) }\end{array}$} & \multirow{2}{*}{$491 \mathrm{bp}$} \\
\hline Bab18S_multiR & 5'-GACGACCTCCAATCACTAGTCGGCATAG-3' & 63.4 & & \\
\hline Hep18S_multiF & 5'-AACGACTCCTTCAGCACCTTACGAGAA-3' & 62.7 & \multirow{2}{*}{ Hepatozoon canis } & \multirow{2}{*}{$376 \mathrm{bp}$} \\
\hline Hep18S_multiR & 5'-TCACAGACCTGTTATTGCCTCAAACTTCCT-3' & 63.4 & & \\
\hline
\end{tabular}


Table 2. Prevalence of Hepatozoon canis, Babesia vogeli, Ehrlichia canis, and Anaplasma platys infections and the type and number of symptoms found in hosts

\begin{tabular}{|c|c|c|c|c|}
\hline & Hepatozoon canis & Babesia vogeli & Ehrlichia canis & Anaplasma platys \\
\hline Prevalence & $6 / 114(5.26 \%)$ & $6 / 114(5.26 \%)$ & $6 / 114(5.26 \%)$ & $4 / 114(3.51 \%)$ \\
\hline \multicolumn{5}{|l|}{ Symptoms } \\
\hline High fever $\left(>39^{\circ} \mathrm{C}\right)$ & $0 / 6$ & $0 / 6$ & $0 / 6$ & $0 / 4$ \\
\hline Low platelet count $\left(<200,000\right.$ platelets $\left./ \mathrm{mm}^{3}\right)$ & $1 / 6$ & $4 / 6$ & $4 / 6$ & $4 / 4$ \\
\hline Low white blood cell count $\left(<6,000 \mathrm{WBC} / \mathrm{mm}^{3}\right)$ & $0 / 6$ & $0 / 6$ & $0 / 6$ & $0 / 4$ \\
\hline Presence of clinical signs (e.g., lethargy) & $0 / 6$ & $0 / 6$ & $0 / 6$ & $0 / 4$ \\
\hline Presence of ticks & $2 / 6$ & $0 / 6$ & $0 / 6$ & $0 / 4$ \\
\hline Positive serology results for Ehrlichia sp. & $4 / 6$ & $6 / 6$ & $6 / 6$ & $1 / 4$ \\
\hline \multicolumn{5}{|l|}{ Number of symptoms } \\
\hline 1 symptom & $5 / 6$ & $2 / 6$ & $2 / 6$ & $3 / 4$ \\
\hline 2 symptoms & $1 / 6$ & $4 / 6$ & $4 / 6$ & $1 / 4$ \\
\hline 3 symptoms & $0 / 6$ & $0 / 6$ & $0 / 6$ & $0 / 4$ \\
\hline
\end{tabular}

Table 3. DNA sequences of amplicons deposited in GenBank for primers Hep-F/Hep-R (Hepatozoon canis), PIRO-A1/PIRO-B (Babesia vogeli), EHR16SD/EHR16SR (Anaplasmataceae), and fD1/EHR16SR and EHR16SD/Rp2 (Anaplasma platys)

\begin{tabular}{|c|c|c|c|c|}
\hline Sample & $\begin{array}{c}\text { Percent } \\
\text { identification }\end{array}$ & $\begin{array}{l}\text { Percent } \\
\text { coverage }\end{array}$ & $\begin{array}{l}\text { GenBank accession number } \\
\text { of most similar sequence }\end{array}$ & $\begin{array}{c}\text { GenBank accession } \\
\text { number }\end{array}$ \\
\hline EEEE_HepF & 99 & 99 & Hepatozoon canis clone 7243 (KC138535) & KP182929 \\
\hline JJJ_HepF & 99 & 100 & Hepatozoon canis clone 7243 (KC138535) & KP182930 \\
\hline LLL_HepF & 100 & 100 & Hepatozoon canis clone 7243 (KC138535) & KP182931 \\
\hline M_HepF & 99 & 100 & Hepatozoon canis clone 7243 (KC138535) & KP182932 \\
\hline UUUU_HepF & 99 & 100 & Hepatozoon canis clone 7243 (KC138535) & KP182933 \\
\hline VVVV_HepF & 99 & 100 & Hepatozoon canis clone 7243 (KC138535) & KP182934 \\
\hline C_PIRO & 100 & 100 & Babesia canis vogeli SK-011 (JX112785) & KP182935 \\
\hline EEEE_PIRO & 100 & 99 & Babesia canis vogeli SK-011 (JX112785) & KP182936 \\
\hline FFFF_PIRO & 99 & 99 & Babesia canis vogeli SK-011 (JX112785) & KP182937 \\
\hline GGGG_PIRO & 100 & 100 & Babesia canis vogeli SK-011 (JX112785) & KP182938 \\
\hline JJJ_PIRO & 100 & 100 & Babesia canis vogeli SK-011 (JX112785) & KP182939 \\
\hline SSS_PIRO & 99 & 98 & Babesia canis vogeli SK-011 (JX112785) & KP182940 \\
\hline EEE_Ecanis & 99 & 99 & Ehrlichia canis isolate TrKysEcan3 (KJ513197) & KP182941 \\
\hline HHH_Ecanis & 99 & 99 & Ehrlichia canis isolate TrKysEcan3 (KJ513197) & KP182942 \\
\hline LLL_Ecanis & 99 & 100 & Ehrlichia canis isolate TrKysEcan3 (KJ513197) & KP182947 \\
\hline $\mathrm{T}_{-}$Ecanis & 97 & 97 & Ehrlichia canis isolate S3b (KJ659037) & KP182948 \\
\hline UU_Ecanis & 99 & 99 & Ehrlichia canis isolate TrKysEcan3 (KJ513197) & KP182949 \\
\hline YY_Ecanis & 99 & 99 & Ehrlichia canis isolate TrKysEcan3 (KJ513197) & KP182950 \\
\hline HHHH_Anaplasma & 99 & 99 & Uncultured Anaplasma sp. (FJ943580) & KP182943 \\
\hline IIII_Anaplasma & 99 & 99 & Uncultured Anaplasma sp. (FJ943580) & KP182944 \\
\hline QQQ_Anaplasma & 99 & 99 & Uncultured Anaplasma sp. (FJ943580) & KP182945 \\
\hline RRR_Aphagocytophilum & 99 & 97 & Anaplasma phagocytophilum (HG916767) & KP182946 \\
\hline Isolate_RRR & 99 & 97 & Anaplasma platys (AY530806) & KX447502 \\
\hline Isolate_HHHH & 100 & 99 & Anaplasma platys Gigio (EU439943) & KX447503 \\
\hline Isolate_IIII & 99 & 99 & Anaplasma platys isolate A.pl.\#87 (JQ396431) & KX447504 \\
\hline Isolate_QQQ & 100 & 99 & Anaplasma platys isolate A.pl.\#87 (JQ396431) & KX447505 \\
\hline
\end{tabular}

mon signs of infection observed were presence of anti-Ehrlichia IgG antibodies in serum $(71.05 \%)$, low platelet count
(47.37\%), and presence of ticks (14.91\%). The following signs of infection were found in less than $5 \%$ of the dogs: 
presence of clinical signs (3.51\%), high fever (2.63\%), and low white blood cell count $(1.75 \%)$.

\section{PCR detection and DNA sequencing}

PCR and sequencing detected E. canis in $5.26 \%(6 / 114)$, B. vogeli in $5.26 \%(6 / 114), H$. canis in $5.26 \%(6 / 114)$, and $A$. platys in $3.51 \%$ (4/114) out of 114 dogs. All dogs positive for CVBDs exhibited either one or two symptoms (Table 2) whereas all dogs positive for any of the four have either or both low platelet counts and presence of anti-Ehrlichia $\mathrm{sp}$. IgG. Besides, none of the dogs that showed positive infection in PCR and multiplex PCR manifested clinical signs of the disease, high fever, or low white blood cell counts. Lastly, two dogs infected with $H$. canis have ticks found in their fur. All sequences were $97-100 \%$ similar to their respective species identity (Table 3 ). Whilst $H$. canis, B. vogeli, and $E$. canis sequences were $99-100 \%$ similar to their respective identified species. For the Anaplasma sp., the use of EHR16SD/ EHR16SR set of primers exhibited that three of the four samples were $99 \%$ similar to uncultured Anaplasma sp. while the remaining was $97 \%$ similar to A phagocytophilum. Subsequent PCR using fD1/EHR6R and EHR16SD/Rp2 sets of primers to amplify larger parts of the $16 \mathrm{~S}$ rRNA gene, and sequence analysis of products confirmed that the isolates were $97-99 \%$ similar to $A$. platys.

Phylogenetic trees were constructed to confirm species identity of $H$. canis, B. vogeli, and A. platys. Samples clustered with their respective species identity with high bootstrap support from the ML, NJ, and MP phylogenetic trees further confirmed the identity of the samples as H. canis (Fig. 1), B. vogeli (Fig. 2), and A. platys (Fig. 3). The sequences obtained in this study were stored in GenBank with accession nos. KP182929-KP182934 for partial 18S rRNA gene sequences of $H$. canis, KP182935-KP182940 for partial $18 \mathrm{~S}$ rRNA gene sequences of B. vogeli, KP182941-KP182950 for partial 16S rRNA gene sequences of all Anaplasmataceae, and KX447502-KX447505 for full 16S rRNA gene sequences of $A$. platys.

\section{Multiplex PCR}

The multiplex PCR primers were able to amplify their respective targets at all temperatures used in the gradient PCR experiment with lower limits of detection of $4.5 \mathrm{ng} / \mu \mathrm{L}$, $19.1 \mathrm{ng} / \mu \mathrm{L}$, and $2.4 \mathrm{ng} / \mu \mathrm{L}$ for $H$. canis, B. canis, and $E$. canis DNA, respectively (data not shown). To standardize, an annealing temperature of $60^{\circ} \mathrm{C}$ was used for all experiments. The three primer sets amplified their intended target CVB pathogen in both single reaction and multiplex PCR without crossreacting with any of the other bacterial and CVB pathogen DNA used in the analytical specificity study (Fig. 4). Multiplex PCR detected $4.39 \%(5 / 114) H$. canis infections, $5.26 \%$ (6/114) B. vogeli infections, and 5.26\% (6/114) E. canis infections from the 114 dogs. All samples positive for single reaction PCR and sequencing were also positive for multiplex PCR except for one $H$. canis infection. Moreover, multiplex PCR also detected one case of $E$. canis and $H$. canis coinfection and two cases of $B$. vogeli and $H$. canis co-infections.

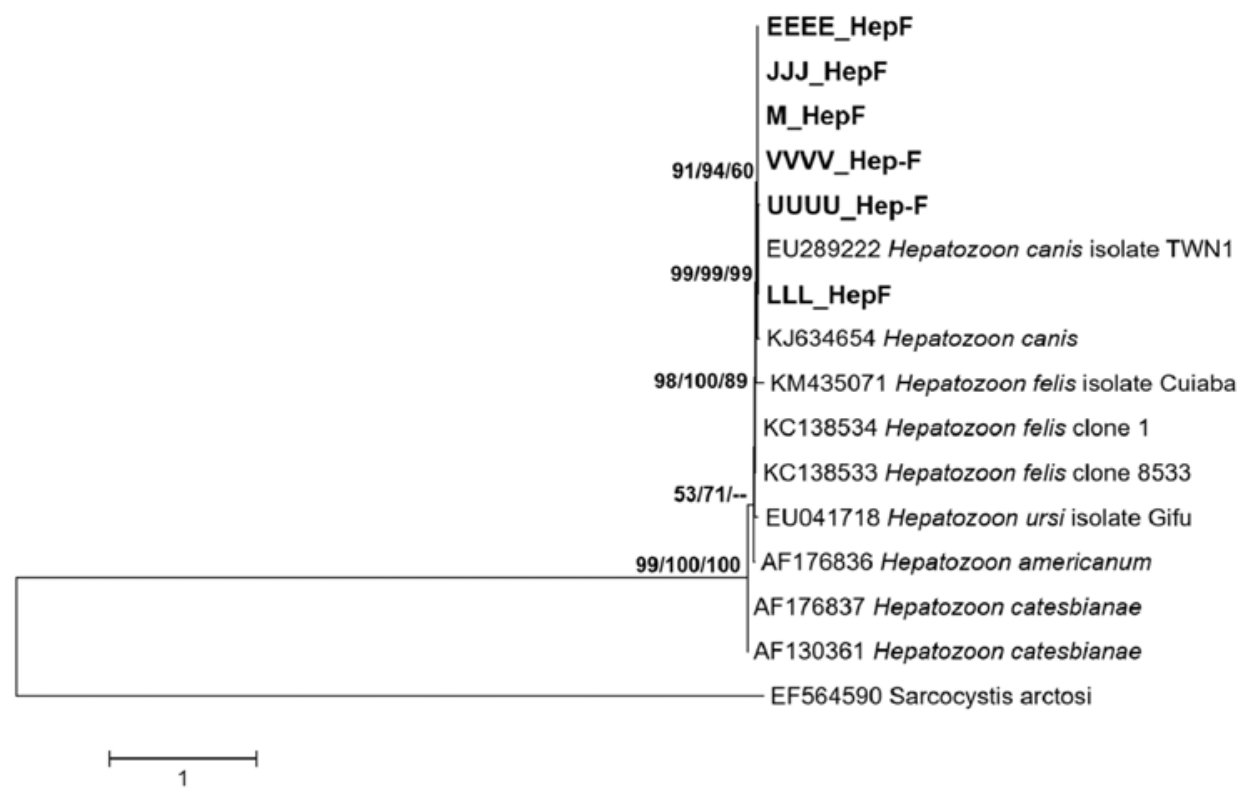

Fig. 1. Consensus phylogenetic tree based on the maximum likelihood (ML) tree using partial 18S rRNA gene sequences (517 unambiguously aligned nucleotide positions) of 15 Hepatozoon specimens and 1 outgroup species (Sarcocystis arctosi). The bootstrap consensus tree was constructed using the HKY model and inferred from 1,000 replicates. The bootstrap values of the three phylogenetic methods used are shown in the order ML/neighbor joining (NJ) and maximum parsimony (MP). The interior branches are marked with if the bootstrap value is less than $50 \%$. 


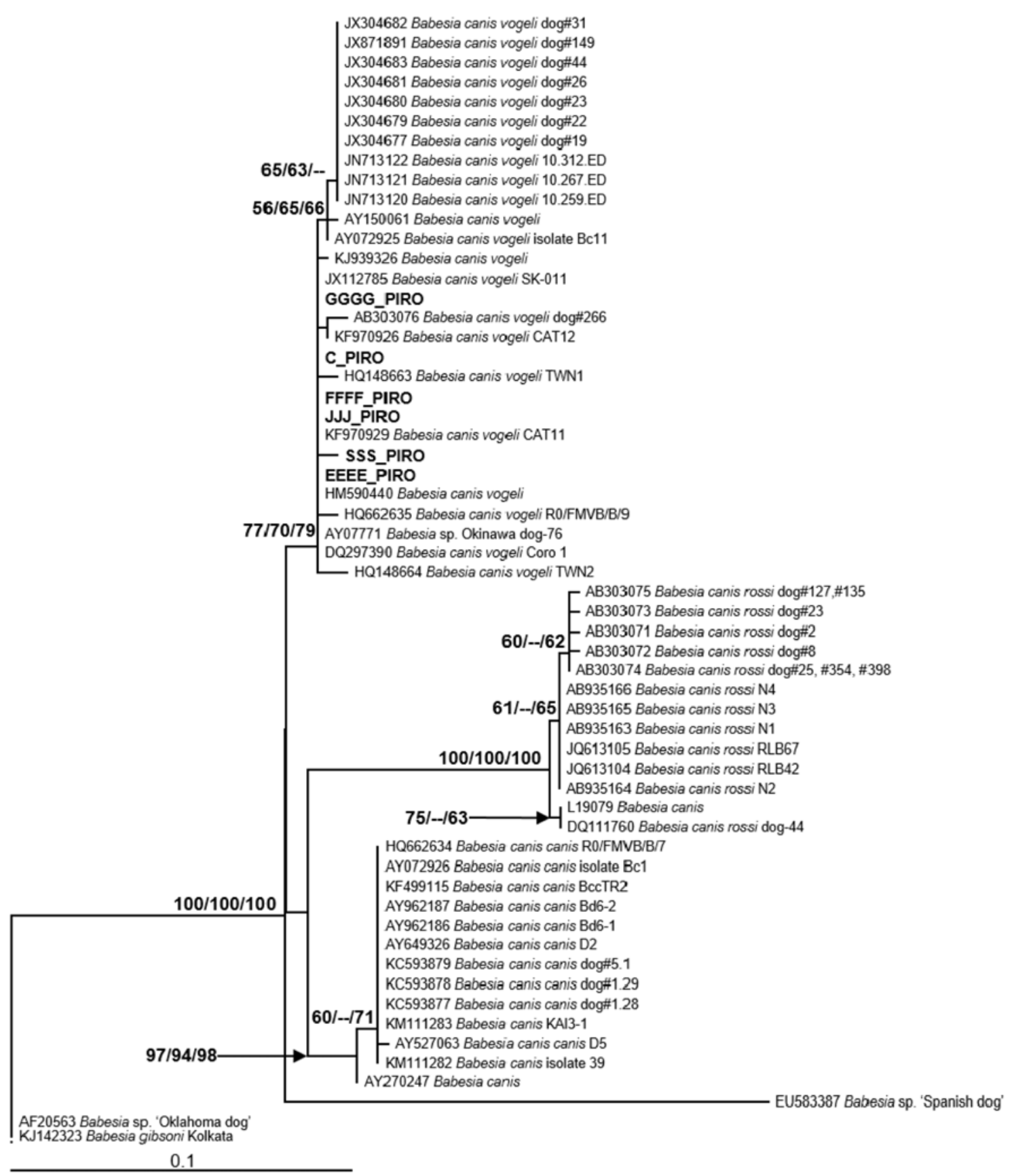

Fig. 2. Consensus phylogenetic tree based on the maximum likelihood (ML) tree using partial 18S rRNA gene sequences (343 unambiguously aligned nucleotide positions) of 56 Babesia specimens and 2 outgroup species (Babesia sp. Oklahoma dog and Babesia gibsoni). The bootstrap consensus tree was constructed using the GTR $+\mathrm{G}$ model and inferred from 1,000 replicates. The bootstrap values of the three phylogenetic methods used are shown in the order ML/neighbor joining (NJ) and maximum parsimony (MP). The interior branches are marked with - if the bootstrap value is less than $50 \%$.

\section{Discussion}

H. canis, B. vogeli, E. canis, and A. platys infections in dogs in Metro Manila, Philippines are present in low prevalence. In this study, H. canis, B. vogeli, E. canis and A. platys were found in $5.26 \%, 5.26 \%, 5.26 \%$, and $3.51 \%$, respectively, of 114 dogs admitted at the Makati Dog and Cat Hos- pital and the Carlos Veterinary Clinic. Phylogenetic analyses and BLAST results confirm the identification of these with high bootstrap support. Notably, the detected Anaplasma infections were identified as $A$. platys after full $16 \mathrm{~S}$ rRNA gene sequencing and phylogenetic analyses. Results show that CVB pathogens in dogs from Metro Manila are more diverse than previously reported. In fact, a previous study 


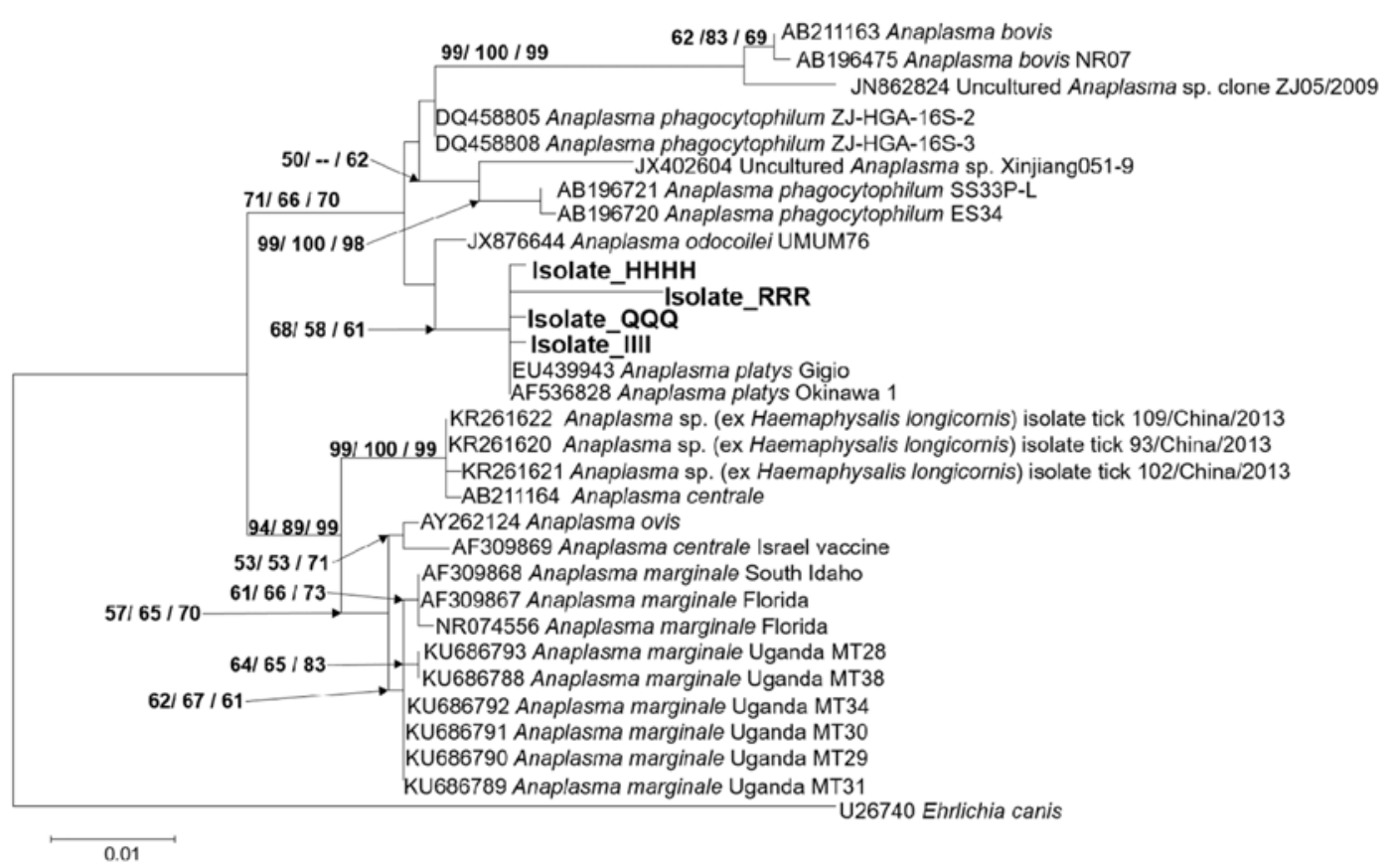

Fig. 3. Consensus phylogenetic tree based on the maximum likelihood (ML) tree using full 16S rRNA gene sequences (819 unambiguously aligned nucleotide positions) of 30 Anaplasma specimens and 1 outgroup species (Ehrlichia canis). The bootstrap consensus tree was constructed using the TrN model and inferred from 1,000 replicates. The bootstrap values of the three phylogenetic methods used are shown in the order ML/neighbor joining (NJ) and maximum parsimony (MP). The interior branches are marked with - if the bootstrap value is less than $50 \%$.
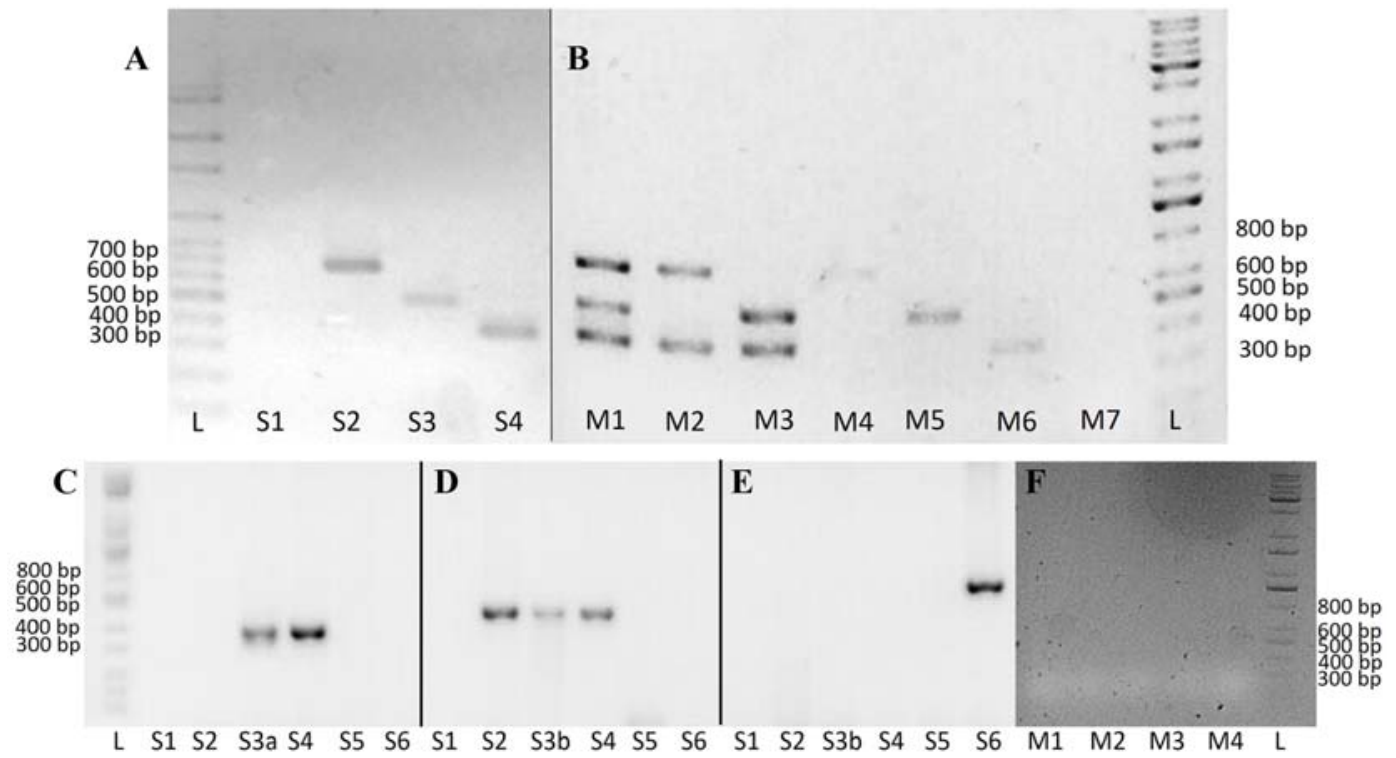

Fig. 4. Results of single reaction and multiplex polymerase chain reaction (PCR) in 1.5\% agarose gel on positive and negative control DNA using the designed multiplex PCR primers. Positive bands are indicated by $376 \mathrm{bp}$ (Hepatozoon [H.] canis), 491 bp (Babesia sp.), and 664 bp (Ehrlichia [E.] canis). (A) Single reaction PCR. L, molecular weight ladder; S1, negative control; S2, E. canis with Ehr16S_multi primer pair; S3, Babesia (B.) canis with Bab18S_multi primer pair; S4, H. canis with Hep18S_multi primer pair. (B) Multiplex PCR. M1, positive control DNA of E. canis, B. canis, and H. canis; M2, mixed DNA sample of E. canis and H. canis; M3, mixed DNA sample of $B$. vogeli and $H$. canis; M4, DNA sample of E. canis; M5, DNA sample of B. vogeli; M6, DNA sample of $H$. canis; M7, negative control; L, molecular weight ladder. Single reaction PCR using primers (C) Hep18S-multi primer pair, (D) Bab18S_multi primer pair, and (E) Ehr16s_multi primer pair: S1, negative control; S2, B. canis; S3a, H. canis; S3b, B. vogeli; S4, B. vogeli and H. canis; S5, A. platys; S6, E. canis; L, molecular weight ladder. (F) Multiplex PCR: M1, negative control; M2, Bacillus subtilis; M3, Staphylococcus aureus; M4, Pseudomonas aeruginosa; M5, Escherichia coli; L, molecular weight ladder. 
only detected one case of canine $H$. canis infection out of 168 blood samples from dogs around Metro Manila using the primers Babesia-F/Babesia-R and blood smear examination [2]. PCR detection has been instrumental in detection of CVB pathogens in the country including published accounts of canine infections of E. canis [36], A. platys [37], and $B$. vogeli [39] in Cebu City and Babesia sp. and E. canis in the province of Nueva Ecija [8]. In addition, dogs seropositive for $B$. gibsoni infections have also been reported in the province of Cavite [12]. More surveys in stray and pet dogs can clarify the prevalence and diversity of CVB pathogens in the country as well as the inherent risks. In neighboring Southeast Asian countries, more than $10 \%$ of sampled population of dogs can be infected with CVB pathogens. For example, a survey of CVBDs out of 101 free-roaming dogs in Cambodia reported prevalences of up to $32.7 \%$ for Babesia spp., $10.9 \%$ for $H$. canis, and $21.8 \%$ for E. canis [14]. Similarly, E. canis prevalence can be as high as $25.5 \%$ of stray dogs in Malaysia [19] and $20.4 \%$ of pet dogs [30] and $21.5 \%$ of stray dogs [27] in Thailand. Moreover, surveys of CVBDs in the Philippines are limited to detection of Hepatozoon spp., Babesia spp., Anaplasma spp., and Ehrlichia spp. Recent studies have already shown presence of Dirofilaria immitis and Mycoplasma spp. in dogs from Cambodia [14] and Mycoplasma spp. in dogs from Thailand [20].

Multiplex PCR combines the sensitivity and specificity of PCR with the added advantage of simultaneous detection of multiple targets in one reaction. The current standard for CVBD detection - blood smear examination with Giemsa staining - has been demonstrated to achieve only up to $74 \%$ sensitivity in detecting $E$. canis after examination of 1,000 oil immersion field slides of both canine buffy coats and lymph nodes [23]. Moreover, detection by blood smear examination becomes difficult in subclinical cases where parasitemia is low [15]. PCR detection in canine blood samples has higher sensitivity compared to blood smear examination which is conventionally used for routine detection of CVBDs [5]. Moreover, multiplex PCR has been proven useful in simultaneous detection of CVBDs since current results show that reliance only on serology and presence of symptoms may not be accurate for diagnosis [18]. Presence of anti-Ehrlichia IgG and low platelet count were the two most commonly observed symptoms in this group of dogs. There were 81 out of 114 dogs positive for serology but only 6 out of the 81 were actually infected with $E$. canis indicating more cases of past infections rather than current ones. Poor correlation or concordance between PCR prevalence and seroprevalence has been recorded before [22, 30, 35] since presence of antibody does not necessarily mean presence of the parasite in blood samples. Low platelet count is a symptom of canine monocytic ehrlichiosis [29] and canine babesiosis [15] and this symptom has been suggested as a screening test for canine monocytic ehrlichiosis to further improve reliability of diagnosis [4]. However, later studies show no correlation with $E$. canis, A. platys, and/or Babesia spp. infections [17, 21]. In this study, there were 54 out of the 114 dogs surveyed that had low platelet counts but only 13 of 54 were infected with any of the four causative agents of CVBDs indicating other causes of thrombocytopenia. Results of conventional PCR and sequencing confirm the results of multiplex PCR. All samples detected by the former methods were also detected using multiplex PCR except for one case of $H$. canis infection. This may be due to a lower limit of detection of Hep-F/ Hep-R compared to Hep18S_multiF/Hep18S_mutiR. Nevertheless, results still indicate the utility of the designed multiplex PCR primers in screening for CVBDs and as a possible aid in diagnosis. The high analytical sensitivity and specificity of the primers are a good compliment for other diagnostic methods such as observation of symptoms, serology, and microscopy since these have known issues in these areas. However, additional tests are necessary to determine the clinical sensitivity and specificity of the primers in larger sample populations due to the low prevalence of CVBDs in the current sample population.

In summary, this study gives crucial information on the underreported diversity and prevalence of CVBDs in the Philippines which have been lacking from 1977 to 2008. In addition, the multiplex PCR protocol developed in this study shows promise in clinical application for screening of CVBDs in canine blood. A proper screening method is necessary for both clinical use and prevalence studies in the Philippines. Future prevalence studies can address the current extent and effect of CVBD as well as determine which species are already present in local dog populations from other parts of the country. The multiplex PCR protocol offers a quick and simultaneous method for screening of CVBDs in local dog populations as well as pets travelling from foreign countries. It is recommended that future studies also add $B$. gibsoni and A. platys to the multiplex detection protocol since these are also present in local canine populations.

\section{Acknowledgments}

We thank Jaime Antonio O. Yu for the technical assistance. We are also grateful to Dr. Shimon Harrus of Israel, Dr. Gabor Foldvari of Hungary, and Dr. Domenico Otranto of Italy for providing DNA extracts of positive controls for E. canis, B. canis, and $H$. canis, respectively. This work was supported by a research grant from the Natural Sciences Research Institute, University of the Philippines Diliman (project code: BIO-14-2-04).

\section{References}

1. Baneth G, Mathew JS, Shkap V, Macintire DK, Barta JR, Ewing SA. Canine hepatozoonosis: two disease syndromes caused by separate Hepatozoon spp. Trends Parasitol 2003, 19, 27-31.

2. Baticados AM, Baticados WN, Carlos ET, Carlos SMEAS, Villarba LA, Subiaga SG, Magcalas JM. Parasitological detection and molecular evidence of Hepatozoon canis from 
canines in Manila, Philippines. Vet Med Res Rep 2010, 1, 710.

3. Brecher ME, Hay SN. Bacterial contamination of blood components. Clin Microbiol Rev 2005, 18, 195-204.

4. Bulla C, Takahira RK, Araújo JP Jr, Aparecida Trinca L, Souza Lopes R, Wiedmeyer CE. The relationship between the degree of thrombocytopenia and infection with Ehrlichia canis in an endemic area. Vet Res 2004, 35, 141146.

5. Cardoso L, Yisaschar-Mekuzas Y, Rodrigues FT, Costa Á, Machado J, Diz-Lopes D, Baneth G. Canine babesiosis in northern Portugal and molecular characterization of vectorborne co-infections. Parasit Vectors 2010, 3, 27.

6. Carlos ET, Carlos ER, Calalay FT, Cabiles CC. Babesia canis: clinical observations in dogs in the Philippines. Philipp J Vet Med 1972, 11, 81-91.

7. Carlos ET, Cruz FB, Cabiles CC, Calalay FT, Carlos ER. Hepatozoon sp. in the WBC of a human patient. UP Vet 1971, 15, 5-7.

8. Corales JMI, Viloria VV, Venturina VM, Mingala CN. The prevalence of Ehrlichia canis, Anaplasma platys and Babesia spp. in dogs in Nueva Ecija, Philippines based on multiplex polymerase chain reaction (mPCR) assay. Ann Parasitol 2014, 60, 267-272.

9. Földvári G, Hell E, Farkas R. Babesia canis canis in dogs from Hungary: detection by PCR and sequencing. Vet Parasitol 2005, 127, 221-226.

10. Guindon S, Dufayard JF, Lefort V, Anisimova M, Hordijk W, Gascuel O. New algorithms and methods to estimate maximum-likelihood phylogenies: assessing the performance of PhyML 3.0. Syst Biol 2010, 59, 307-321.

11. Hall TA. BioEdit: a user-friendly biological sequence alignment editor and analysis program for Windows 95/98/ NT. Nucleic Acids Symp Ser 1999, 41, 95-98.

12. Inokuma H, Beppu T, Okuda M, Shimada Y, Sakata Y. Detection of ehrlichial DNA in Haemaphysalis ticks recovered from dogs in Japan that is closely related to a novel Ehrlichia sp. found in cattle ticks from Tibet, Thailand, and Africa. J Clin Microbiol 2004, 42, 1353-1355.

13. Inokuma H, Okuda M, Ohno K, Shimoda K, Onishi T. Analysis of the 18S rRNA gene sequence of a Hepatozoon detected in two Japanese dogs. Vet Parasitol 2002, 106, 265271.

14. Inpankaew T, Hii SF, Chimnoi W, Traub RJ. Canine vector-borne pathogens in semi-domesticated dogs residing in northern Cambodia. Parasit Vectors 2016, 9, 253.

15. Irwin PJ. Canine babesiosis: from molecular taxonomy to control. Parasit Vectors 2009, 2 (Suppl 1), S4.

16. Jefferies R, Ryan UM, Muhlnickel CJ, Irwin PJ. Two species of canine Babesia in Australia: detection and characterization by PCR. J Parasitol 2003, 89, 409-412.

17. Kelly PJ, Lucas H. Failure to demonstrate Babesia, Anaplasma or Ehrlichia in thrombocytopenic dogs from St Kitts. J Infect Dev Ctries 2009, 3, 561-563.

18. Kledmanee K, Suwanpakdee S, Krajangwong S, Chatsiriwech J, Suksai P, Suwannachat P, Sariya L, Buddhirongawatr $\mathbf{R}$, Charoonrut $\mathbf{P}$, Chaichoun $\mathbf{K}$. Development of multiplex polymerase chain reaction for detection of Ehrlichia canis, Babesia spp and Hepatozoon canis in canine blood. Southeast Asian J Trop Med Public
Health 2009, 40, 35-39.

19. Koh FX, Panchadcharam C, Tay ST. Vector-borne diseases in stray dogs in peninsular Malaysia and molecular detection of Anaplasma and Ehrlichia spp. from Rhipicephalus sanguineus (Acari: Ixodidae) ticks. J Med Entomol 2016, 53, 183-187.

20. Liu M, Ruttayaporn N, Saechan V, Jirapattharasate C, Vudriko P, Moumouni PFA, Cao S, Inpankaew T, Ybañez AP, Suzuki H, Xuan X. Molecular survey of canine vectorborne diseases in stray dogs in Thailand. Parasitol Int 2016, $\mathbf{6 5}, 357-361$.

21. Macieira DB, Messick JB, Cerqueira AMF, Freire IMA, Linhares GFC, Almeida NKO, Almosny NRP. Prevalence of Ehrlichia canis infection in thrombocytopenic dogs from Rio de Janeiro, Brazil. Vet Clin Pathol 2005, 34, 44-48.

22. Maggi RG, Birkenheuer AJ, Hegarty BC, Bradley JM, Levy MG, Breitschwerdt EB. Comparison of serological and molecular panels for diagnosis of vector-borne diseases in dogs. Parasit Vectors 2014, 7, 127.

23. Mylonakis ME, Koutinas AF, Billinis C, Leontides LS, Kontos V, Papadopoulos O, Rallis T, Fytianou A. Evaluation of cytology in the diagnosis of acute canine monocytic ehrlichiosis (Ehrlichia canis): a comparison between five methods. Vet Microbiol 2003, 91, 197-204.

24. Novilla MN, Kwapien RP, Peneyra RS. Occurrence of canine hepatozoonosis in the Philippines. Proc Helminthol Soc Wash 1977, 44, 98-101.

25. Otranto D, Dantas-Torres F, Breitschwerdt EB. Managing canine vector-borne diseases of zoonotic concern: part one. Trends Parasitol 2009, 25, 157-163.

26. Parola P, Roux V, Camicas JL, Baradji I, Brouqui P, Raoult D. Detection of ehrlichiae in African ticks by polymerase chain reaction. Trans $\mathrm{R}$ Soc Trop Med Hyg 2000, 94, 707-708.

27. Piratae S, Pimpjong K, Vaisusuk K, Chatan W. Molecular detection of Ehrlichia canis, Hepatozoon canis and Babesia canis vogeli in stray dogs in Mahasarakham province, Thailand. Ann Parasitol 2015, 61, 183-187.

28. Sabino CV, Weese JS. Contamination of multiple-dose vials in a veterinary hospital. Can Vet J 2006, 47, 779-782.

29. Shaw SE, Day MJ, Birtles RJ, Breitschwerdt EB. Tickborne infectious diseases of dogs. Trends Parasitol 2001, 17, 74-80.

30. Suksawat J, Xuejie Y, Hancock SI, Hegarty BC, Nilkumhang P, Breitschwerdt EB. Serologic and molecular evidence of coinfection with multiple vector-borne pathogens in dogs from Thailand. J Vet Intern Med 2001, 15, 453-462.

31. Swofford DL. PAUP*. Phylogenetic analysis using parsimony (*and other methods). Version 4.0b10. Sinauer Associates, Sunderland, 2003.

32. Taboada J, Lobetti R. Babesiosis. In: Green CE (ed.). Infectious Diseases of the Dog and Cat. 3rd ed. pp. 722-736, WB Saunders, Philadelpia, 2006.

33. Tamura K, Stecher G, Peterson D, Filipski A, Kumar S. MEGA6: Molecular Evolutionary Genetics Analysis version 6.0. Mol Biol Evol 2013, 30, 2725-2729.

34. Watanabe M, Oikawa T, Hiraoka H, Kaneko N, Itamoto K, Mizuno T, Okuda M, Inokuma H. Experimental inoculation of beagle dogs with Ehrlichia species detected from Ixodes ovatus. Vet Parasitol 2006, 136, 147-154. 
35. Wong SSY, Teng JLL, Poon RWS, Choi GKY, Chan KH, Yeung ML, Hui JJY, Yuen KY. Comparative evaluation of a point-of-care immunochromatographic test SNAP 4Dx with molecular detection tests for vector-borne canine pathogens in Hong Kong. Vector Borne Zoonotic Dis 2011, 11, 12691277.

36. Ybañez AP. First molecular evidence of Ehrlichia canis infection in dogs with probable disease relapse in the Philippines. J Adv Vet Res 2014, 4, 184-188.
37. Ybañez AP. First report on Anaplasma platys infection in a dog in the Philippines. Iran J Vet Med 2013, 7, 227-231.

38. Ybañez AP, Perez ZO, Gabotero SR, Yandug RT, Kotaro M, Inokuma H. First molecular detection of Ehrlichia canis and Anaplasma platys in ticks from dogs in Cebu, Philippines. Ticks Tick Borne Dis 2012, 3, 288-293.

39. Ybañez AP, Ybañez RHD, Talle MG, Liu M, Moumouni PFA, Xuan X. First report on Babesia vogeli infection in dogs in the Philippines. Parasitol Int 2017, 66, 813-815. 